\title{
Psychosocial adjustment 17 years after severe brain injury
}

\author{
R L Wood, N A Rutterford
}

J Neurol Neurosurg Psychiatry 2006;77:71-73. doi: 10.1136/jnnp.2005.065540

\begin{abstract}
Objectives: To examine very long term psychosocial outcome following severe brain injury in a large cohort, with the aim of evaluating Thomsen's observation that even after very serious head trauma the long term outcome in some patients is reasonably good. Methods: The cohort consisted of 80 patients who had suffered severe brain injury evaluated at a mean time of 17 years post injury (range 10-32 years). Information regarding employment status and relationship status was obtained during clinical interview. Psychosocial outcome measures included the

Supervision Rating Scale, Satisfaction with Life Scale, Hospital Anxiety and Depression Scale (HADS), Patient Competency Rating Scale, and Community Integration Questionnaire.

Results: Of the cohort, $72.0 \%$ lived independently, 28.7\% were in full time employment, and $60.0 \%$ were married or cohabiting. The mean rating of life satisfaction was "slightly dissatisfied", but no serious emotional problems were evident from self report ratings on the HADS. Mean functional competency ratings and community integration levels were just below those reported for non-disabled patients.

Conclusions: Results indicate that although long term psychosocial functioning in patients with severe head injury remains compromised, long term adjustment may be better than expected from data reported by studies assessing psychosocial outcome at earlier stages of recovery.
\end{abstract}

See end of article for authors' affiliations

Correspondence to:

Professor Rodger L Wood,

Department of Psychology, University of Wales

Swansea, Singleton Park,

Swansea SA2 8PP, UK.

r.l.wood@swansea.ac.uk

Received 14February 2005

Revised version received

13 June 2005

Accepted 15 June 2005
$\mathrm{T}$ homsen observed that even after very serious head trauma, the long term outcome for some patients is reasonably good. ${ }^{1}$ However, follow up studies on individuals who suffer severe head injury report poor psychosocial outcome, ${ }^{2-4}$ with little evidence of improvement during the first 7 years post injury. ${ }^{5}$ Nevertheless, there may be improved psychosocial adjustment in later years. The longitudinal research conducted by Thomsen ${ }^{16}$ found that at early stages of recovery, psychosocial sequelae were more socially debilitating than physical disability, increasing the risks of social isolation, caregiver stress, and unemployment. These problems persisted for a number of years following the initial follow up, but late psychosocial improvement became evident in some patients after 10-15 years. ${ }^{1}$ Half of the patients monitored, who needed constant support for several years after the incident, eventually achieved independence and some even regained the capacity to work. In her final 20 year follow up, ${ }^{7}$ Thomsen noted that nearly half of 31 patients $(49.2 \%)$ were capable of living alone, four $(12.5 \%)$ were married, four (12.5\%) continued to live with parents, and eight $(25.8 \%)$ were in a nursing home. Thomsen noted that the range of individual differences in psychosocial outcome could not be explained by severity of injury, so the quality of long term psychosocial outcome may depend more on the time post injury when a follow up study is conducted.

Thomsen explored very long term outcome in a group of 40 very seriously injured patients (post traumatic amnesia (PTA) $>$ l month). The present study aimed to evaluate psychosocial outcome in a less severely brain injured cohort (mean PTA 19.3 days), between 10 and 32 years post injury (mean 17 years). It was hypothesised that if a reasonable number of very severely brain injured patients are capable of late psychosocial improvement, then patients with severe injury should exhibit at least a comparable level of late psychosocial adjustment. Such patients, therefore, would have a better long term prognosis following serious head injury than currently exists based on data from outcome studies conducted at earlier stages of recovery.

\section{METHODS}

\section{Participants}

Seriously brain injured patients were drawn from two sources: (a) the first author's medico-legal archive database $(\mathrm{n}=348)$; and (b) the head injury archive files at Morriston Hospital, Swansea $(n=164)$. All litigation cases had been settled at least 5 years before the time of follow up. All patients had suffered a severe traumatic brain injury, classified by PTA (mean 19.30, median 14, SD 23.21 days) and were at least 10 years post injury (mean 17.20, SD 5.43, range 10.08-32.17 years). PTA duration was ascertained retrospectively, according to the guidelines proposed by McMillan et al, ${ }^{8}$ that is, last memory prior to incident, first memory following the incident, and assessment for return of continuous day-to-day memory. The 362 subjects who satisfied these criteria were contacted by letter and $133(36.74 \%)$ replied. Of these, $80(60.15 \%)$ expressed a willingness to participate (medico-legal group, $\mathrm{n}=44$; Morriston Hospital group, $\mathrm{n}=36$ ) and formed the follow up cohort. Mean age at injury was 30.53 (SD 12.56) years and at follow up, 47.09 (SD 12.49) years. Mean educational level was 11.94 (SD 2.40 ) years and $57(71.3 \%)$ of the group were male.

\section{Design and procedure}

A cross sectional design was employed to collect information on late psychosocial outcome. Participants were interviewed at home, in the company of a close relative, to clarify injury details and obtain information regarding employment and relationship history. After completing a neuropsychological assessment (data collected for a parallel study), participants were instructed on completing a set of questionnaires (the time taken for interview and neuropsychological examination was $4 \mathrm{~h}$, so the questionnaires were

Abbreviations: $\mathrm{ClQ}$, Community Integration Questionnaire; HADS, Hospital Anxiety and Depression Scale; PCRS, Patient Competency Rating Scale; PTA, post traumatic amnesia; SRS, Supervision Rating Scale; SWLS, Satisfaction with Life Scale 
left with the participant to be completed and returned within the following few days). A total of $56(70 \%)$ fully completed sets of questionnaires was returned. Completion rates of individual measures ranged from a minimum of $75.0 \%$ (CIQ) to $80.0 \%$ (HADS). No differences were found between data from the 56 patients who completed all questionnaires and the 80 patients who returned partially completed sets of questionnaires. The results reported are therefore based on the maximum number of completed individual questionnaires.

\section{Measures}

\section{Demographic variables}

Pre and post injury employment status was categorised as: full time employed/education, part time employed, unemployed, and retired. Relationship status was categorised as: married/cohabiting, divorced/separated, single, and widowed.

\section{Outcome variables}

Supervision Rating Scale (SRS)

The SRS is a measure of independent living rated by the second author. A higher rating indicates greater dependence (range 1-13). ${ }^{\text {? }}$

\section{Satisfaction with Life Scale (SWLS)}

The SWLS is a measure of overall subjective well being. A higher rating indicates greater satisfaction (range 5-35). ${ }^{10}$

Patient Competency Rating Scale (PCRS)

The PCRS is used to assess functional competency in the areas of Cognition, Activities of Daily Living, Interpersonal Behaviour, and Emotional Behaviour. ${ }^{11}{ }^{12}$ A higher rating indicates greater competency (range 1-5).

\section{Community Integration Questionnaire (CIQ)}

The CIQ consists of subscales measuring Home Integration (range 0-10), Social Integration (range 0-12), and Productive Activity (range 0-7). A higher rating indicates greater integration (range 0-29). ${ }^{13}$

Hospital Anxiety and Depression Scale (HADS)

A higher rating in the HADS indicates greater levels of anxiety or depression (range $0-21) .{ }^{14}$

\section{RESULTS}

\section{Supervision Rating Scale}

A mean rating of 1.68 (SD 1.94, range $1-10, \mathrm{n}=80$ ) indicates that the majority $(58,72 \%)$ of participants fell within the "independent" section of the scale. None of the cohort were in care or, with the exception of one patient who was seriously physically disabled, required paid support at home.

\section{Employment status}

A total of $23(28.7 \%)$ participants were in full time employment at follow up compared to $73(91.3 \%)$ pre-injury, while $10(12.5 \%)$ were in part time work and $10(12.5 \%)$ had retired, leaving $37(46.3 \%)$ unemployed.

\section{Relationship status}

Only 17 participants $(21.3 \%)$ were single at follow up compared to $38(47.5 \%)$ pre-injury. Those married/cohabiting increased from $(37,46.3 \%)$ pre-injury to $48(60.0 \%)$ at follow up. The proportion divorced or separated at follow up was less than reported in other studies (pre-injury 5, 6.2\%; follow up $12,15.0 \%)$. Three patients were widowed (3.7\%).

\section{Satisfaction with life}

A mean rating of 19.26 (SD 8.28, range $5-35, \mathrm{n}=62$ ) in the SWLS fell just inside the "slightly dissatisfied" range, $^{15}$ consistent with data obtained from a brain injured cohort 10 years post injury. ${ }^{16}$

\section{Anxiety and depression}

A mild anxiety score was recorded (mean 8.48, SD 4.41, range $0-19, \mathrm{n}=64)$. The depression score was in the normal range (mean 6.13, SD 4.20 , range $0-16, \mathrm{n}=64$ ). There was no indication that the group suffered serious emotional problems.

\section{Functional competency}

Mean subscale ratings in the PCRS were as follows: Activities of Daily Living 4.25 (SD 0.73, range 1-5); Cognition 3.67 (SD 0.84, range 1-5); Interpersonal Behaviour 3.63 (SD 0.75, range 2-5); and Emotional Behaviour 3.38 (SD 0.84, range 15). Responses fell between the ratings of: "Can do with some difficulty" and "Fairly easy to do". Hall et al ${ }^{17}$ suggest that these scores fall slightly below the range of scores expected from non-disabled people.

\section{Community integration}

Mean total score in the CIQ was 16.65 (SD 5.22, range 6-28, $\mathrm{n}=60$ ), below that of a group of non-disabled participants (20.71; SD 3.22), ${ }^{18}$ suggesting sub-optimal community integration. Mean subscale scores were: Home Integration 4.85 (SD 2.95, range 0-10); Social Integration 7.63 (SD 2.62, range 0-12); and Productive Activity 4.25 (SD 1.98, range 0$6)$.

\section{DISCUSSION}

These data suggest that very long term psychosocial outcome following serious head injury may be better than expected from data reported at earlier stages of recovery. Seventy two per cent of patients were rated as capable of independent living and $41 \%$ were in either full or part time employment. None were in residential care and only one had care support at home, linked to serious physical disability. Sixty per cent of patients were married or cohabiting and there was a low divorce/separation rate. Most participants rated their functional competency as slightly below that of non-brain injured individuals. This perception may be associated with suboptimal social participation, as measured by the CIQ, an observation also made by Colantonio et $a l^{19}$ at a similar time post injury. The group only described themselves as "slightly dissatisfied" with life, ${ }^{10}$ which might reflect a gradual adjustment to persisting difficulties imposed by brain injury. However, there was no indication that relatively low satisfaction with life ratings translated into psychological morbidity. The group reported mild levels of anxiety but normal ratings of mood, in contrast to Holsinger $e t a l^{20}$ who found long term emotional problems after head injury in war veterans.

The authors acknowledge that responder bias could influence the results of this study as participants and their families consenting to take part might have been those who had made the best personal adjustment. However, we believe the findings support Thomsen's observation that late psychosocial improvement is possible after both severe and very severe brain injury.

\section{Authors' affiliations \\ R L Wood, N A Rutterford, University of Wales, Swansea, UK \\ Competing interests: none declared}

Full ethical approval for this study was obtained from the Department of Psychology, University of Wales, Swansea and the Local Research Ethics Committee of Swansea NHS Trust 


\section{REFERENCES}

1 Thomsen IV. Late outcome of very severe blunt head trauma: a 10-15 year second follow-up. J Neurol Neurosurg Psychiatry 1984;47:260-8.

2 Thornhill S, Teasdale GM, Murray GD, et al. Disability in young people and adults one year after head injury: prospective cohort study. BMJ 2000;320:1631-5.

3 Teasdale GM, Roy C, McMillan TM, et al. The further outcome of hospitalised head injuries. CZH 2004/4/48. Report to the Chief Scientist Office, Edinburgh, UK.

4 Jacobs HE. Adult community integration. In: Bach-y-Rita P, eds. Traumatic brain injury. New York: Demos, 1989:287-318.

5 Oddy M, Coughlan T, Tyerman A, et al. Social adjustment after closed head injury: a further follow-up seven years after injury. J Neurol Neurosurg Psychiatry 1985;48:564-8.

6 Thomsen IV. The patient with severe head injury and his family. Scand J Rehabil Med 1974;6:180-3.

7 Thomsen IV. Late psychosocial outcome in severe traumatic brain injury. Scand J Rehabil Med Suppl 1992;26:142-52.

8 McMillan TM, Jongen ELMM, Greenwood RJ. Assessment of post-traumatic amnesia after severe closed head injury: retrospective or prospective? J Neurol Neurosurg Psychiatry 1996;60(4):422-7.

9 Boake C. Supervision Rating Scale: a measure of functional outcome from brain injury. Arch Phys Med Rehabil 1996;77(8):765-72.

10 Diener E, Emmons RA, Larsen RJ, et al. The Satisfaction with Life Scale. J Pers Assess 1985;49(1):71-5
11 Prigatano GP, Altman IM. Impaired awareness of behavioural limitations after traumatic brain injury. Arch Phys Med Rehabil 1990;71:1058-64.

12 Leathem JM, Murphy $\sqcup$, Flett RA. Self- and informant ratings on the Patien Competency Rating Scale in patients with traumatic brain injury. J Clin Exp Neuropsychol 1998;20(5):694-705.

13 Willer B, Linn RT, Allen K. Community integration and barriers to integration for individuals with brain injury. In: Finlayson MAJ, Garner S, eds. Brain injury rehabilitation: clinical considerations. Baltimore: Williams and Wilkins, 1993:355-75.

14 Zigmond AS, Snaith RP. The Hospital Anxiety and Depression Scale. Acta Psychiatr Scand 1983;67:361-70.

15 Pavot W, Diener E. Review of the Satisfaction with Life Scale. Psychol Assess 1993;5(2): 164-72.

16 Brzuzy S, Speziale BA. Persons with traumatic brain injuries and their families: living arrangements and well-being post injury. Soc Work Health Care 1997;26:77-88.

17 Hall KM, Bushnik T, Lakisic-Kazaic B, et al. Assessing traumatic brain injury outcome measures for long-term follow-up of community based individuals. Arch Phys Med Rehabil 2001:82:367-74.

18 Willer B, Ottenbacher KJ, Coad ML. The Community Integration Questionnaire: a comparative examination. Am J Phys Med Rehabil 1994;73(2):103-11

19 Colantonio A, Ratcliff G, Chase S, et al. Long term outcomes after moderate to severe traumatic brain injury. Dis Rehabil 2004;26(5):253-61.

20 Holsinger T, Steffens DC, Phillips $C$, et al. Head injury in early adulthood and the lifetime risk of depression. Arch Gen Psychiatry 2002;59(1):17-22.

\section{NEUROLOGICAL PICTURE}

\section{Bilateral hypertrophic olivary degeneration}

A 39 years old woman was admitted to neurological department after acute onset of right haemiparesis, vertigo, and oculomotor paresis Magnetic resonance (MR) disclosed multiple ischaemic lesions in the brainstem involving pons and mesencephalon, and altered signal in the basilar artery corresponding to partial trombosis. The patient developed coma, was admitted to the ICU, and treated with anticoagulant drugs. Her clinical condition gradually improved: after 1 month she was alert, dysartric, there was bilateral dysmetria of the arms without paresis and a bilateral orizzontal gaze paresis with dissociated nystagmus (one and half syndrome), and no palatal myoclonus was present. A second MR was performed: no findings of basilar occlusion were present, ischaemic damage was evident in the pons and in the median region of the midbrain. An altered bilateral and symmetric signal in the inferior olivar nucleus was also evident (increased signal on T2 and proton density images) (see figure).

Hypertrophic olivary degeneration is a rare finding secondary to focal lesions of the brain stem involving a functional system-the Guillain-Mollaret triangle-composed of the contralateral dentate nucleus, the ipsilateral red nucleus, the ipsilateral central tegmental tract, and the ipsilateral inferior olivary nucleus.

In the CNS the degeneration of an anatomical structure is usually characterised by neuronal loss replaced by proliferation of glial elements. Unique to the inferior olivary nucleus is transneuronal degeneration resulting in hypertrophy of the targeted region. Pathologically, cell body enlargement, vacuolation of the cytoplasm, astrocytic hyperplasia and proliferation, demyelination, and fibrillary gliosis have been described. The palatal myoclonus and other involuntary movements are the hallmark symptom of this disease and have been described since 1886 .

Most commonly a lesion involving central tegmental tract causes unilateral olivary degeneration. We consider our patient having bilateral hypertrophic degeneration from a unique ischaemic lesion located in the midbrain and corresponding to region of Werneking decussation (decussation of the superior cerebellar peduncle or brachium conjunctivum). Central tegmental tract, arising from the parvocellular part of red nucleus, cannot be damaged by a unique lesion located in mesencephalic tegmentum. The same lesion involving bilateral cerebello-rubro fibers explains bilateral cerebellar ataxia of the arms.

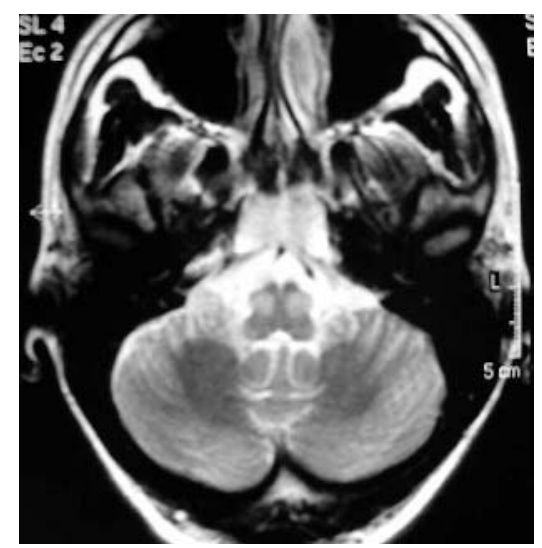

The differential diagnosis of signal hyperintensity on $\mathrm{T} 2$ weighted images within the pontomedullary region includes tumours, demyelinating lesions, infarction, and inflammatory processes. The lack of contrast enhancement, however, is against many tumourous entities or an infectious origin. Therefore, a T2 hyperintense non-contrast enhancing lesion that is accompanied by enlargement of the olivary nucleus, particularly if bilateral and simmetric, as in this patient, can be explained only by hypertrophic olivary degeneration; typically MR hyperintensity was evident after one month, the signal hyperintensity was absent in the first neuroradiological examination. In the next few months the patient could complain palatal myoclonus that is not evident now and has been described until one year after initial damage.

C Gerace, M R Fele, R Luna, G Piazza Azienda Ospedaliera S.Camillo-Forlanini, Rome, Italy

Correspondence to: C Gerace, Neuroscience Department, Azienda Ospedaliera S.CamilloForlanini, Rome, Italy; C.Gerace@tiscalinet .it

\section{References}

1 Krings T, Foltys H, Meister IG, et al. Hypertrophic olivary degeneration following pontine haemorrhage: hypertensive crisis or cavernous haemangioma bleeding? J Neurol Neurosurg Psychiatry 2003;74:797-9.

2 Salamon-Murayama N, Russell EJ, Rabin BM Diagnosis please. Case 17: hypertrophic olivary degeneration secondary to pontine haemorrhage, Radiology 1999;213:81417.

3 Goyal M, Versnick E, Tuite P, et al. Hypertrophic olivary degeneration: metaanalysis of the temporal evolution of MR findings. Am J Neuroradiol 2000:21:1073-7. 